MATHEMATICS OF COMPUTATION

Volume 71, Number 237, Pages 125-145

S $0025-5718(01) 01330-8$

Article electronically published on July 22, 2001

\title{
BACKWARD EULER DISCRETIZATION OF FULLY NONLINEAR PARABOLIC PROBLEMS
}

\author{
C. GONZÁlez, A. OSTERMAnN, C. PALENCIA, AND M. THALHAMMER
}

\begin{abstract}
This paper is concerned with the time discretization of nonlinear evolution equations. We work in an abstract Banach space setting of analytic semigroups that covers fully nonlinear parabolic initial-boundary value problems with smooth coefficients. We prove convergence of variable stepsize backward Euler discretizations under various smoothness assumptions on the exact solution. We further show that the geometric properties near a hyperbolic equilibrium are well captured by the discretization. A numerical example is given.
\end{abstract}

\section{Introduction}

Within the past several years, nonlinear evolution equations of parabolic type have attracted a lot of interest, both in theory and applications. This is due to the fact that such equations are increasingly used for the description of processes involving nonlinear diffusion or heat conduction. As examples we mention reactiondiffusion equations that arise in combustion modeling, the Bellman equations from stochastic control and the nonlinear Cahn-Hilliard equation from pattern formation in phase transitions. Further examples are semilinear problems with moving boundaries, such as the Stefan problem that describes the melting of ice.

The knowledge about stability and convergence for time discretizations of nonlinear parabolic problems has also increased considerably. For Runge-Kutta discretizations of semilinear problems, asymptotically sharp error bounds are given in [9]. Optimal convergence results for quasilinear problems in Hilbert spaces can be found in [10, whereas the papers [5] and [13] deal with stability and convergence of quasilinear problems in Banach spaces. Convergence of linearly implicit RungeKutta methods for nonlinear parabolic problems is studied in [1]; corresponding results for multistep discretizations can be found in [1] and [8]. For the fully nonlinear situation, however, not that much is known. A reason for this might be that the analytical frameworks for fully nonlinear equations are often quite involved.

The present paper is based on a new and simple framework, given in [12, that extends ideas from the semilinear case to the fully nonlinear one. This is done as

Received by the editor January 6, 2000.

2000 Mathematics Subject Classification. Primary 65M12, 65M15; Secondary 35K55, 35R35, 65L06, 65L20.

Key words and phrases. Nonlinear parabolic problems, time discretization, backward Euler method, convergence estimates, stability bounds, asymptotic stability, hyperbolic equilibrium.

The authors acknowledge financial support from Acciones Integradas Hispano-Austríacas 1998/99.

(C)2001 American Mathematical Society 
follows. Consider a parabolic evolution equation

$$
u^{\prime}=F(u), \quad t>0, \quad u(0) \text { given, }
$$

on a Banach space $X$. The nonlinearity $F$ is defined on an open subset $\mathcal{D}$ of a second Banach space $D \subset X$ and takes values in $X$. By linearizing $F$ around a state $u^{*} \in \mathcal{D}$, equation (1.1) takes the form of a semilinear problem

$$
u^{\prime}=A u+f(u), \quad t>0, \quad u(0) \in \mathcal{D},
$$

where $A$ is a bounded operator from $D$ to $X$. Under the assumption that $A$ generates an analytic semigroup, we have a (formal) representation of the solution $u$ by the variation-of-constants formula

$$
u(t)=\mathrm{e}^{t A} u(0)+\int_{0}^{t} \mathrm{e}^{(t-\tau) A} f(u(\tau)) \mathrm{d} \tau, \quad 0 \leq t \leq T .
$$

Since $f(u(t))$ is only defined for $u(t) \in \mathcal{D}$, we have to consider the semiflow in $D$. But as the analytic semigroup $\mathrm{e}^{t A}: X \rightarrow D$ behaves like $C t^{-1}$, the integral on the right-hand side might not exist in $D$. Consequently (1.2) cannot be used directly.

This is quite different to the semilinear case where intermediate spaces $V$ between $X$ and $D$ are considered. There, under the assumption that the function $f$ is locally Lipschitz continuous from $V$ to $X$, a unique local solution can be constructed by a fixed-point iteration relying on formula [1.2) (see [7] and [15]).

It turns out that the following slight modification of the variation-of-constants formula

$$
u(t)=\mathrm{e}^{t A} u(0)+\int_{0}^{t} \mathrm{e}^{(t-\tau) A}(f(u(\tau))-f(u(t))) \mathrm{d} \tau+\int_{0}^{t} \mathrm{e}^{\tau A} \mathrm{~d} \tau f(u(t))
$$

is the basic tool for the analysis of fully nonlinear equations. Within the space of $\alpha$-Hölder continuous functions this relation has a precise meaning and is used to prove existence and uniqueness of a local solution (see [12, Section 8]).

The aim of the present paper is to derive existence and convergence results for time discretizations of (1.1). To keep this exposition in a reasonable length and to avoid technical details, we restrict our attention to the backward Euler method, but we allow variable stepsizes. The extension to strongly $A(\phi)$-stable Runge-Kutta methods with constant stepsizes will be given in [17]. To our knowledge, this is the first paper that provides rigorous error bounds for variable stepsize discretizations of nonlinear parabolic problems. The proofs are based on a global representation of the numerical method by means of a discrete variation-of-constants formula similar to (1.3).

In Section 2 we give the precise assumptions on the initial value problem (1.1) and we present two examples of nonlinear parabolic initial-boundary value problems that fit into this analytical framework. Besides, we introduce spaces of $\alpha$-Hölder continuous sequences on which our discrete framework is based.

Section 3 deals with the existence and uniqueness of the numerical solution, and with convergence. More precisely, we prove in Theorem 3.3 the expected convergence of order one for constant stepsize discretizations of sufficiently smooth solutions. For variable stepsizes and/or less regular solutions, we show convergence of reduced order.

In Section 4 we study the question whether the dynamics of the analytical problem is well captured by the discretization. As an illustration, we consider exponentially stable equilibria and show that the numerical solution locally exists for 
all positive times and decays exponentially towards the equilibrium. A numerical experiment that is in line with our theoretical result is presented.

The auxiliary results for Sections 3 and 4 are finally given in Section 5

\section{AnAlytical FRAMEWORK AND EXAMPLES}

In this section we give the precise hypotheses for (1.1) and further introduce some notation that will be used throughout the paper.

We work in the analytical framework given by [12]. Let $(X,\|\cdot\|)$ and $\left(D,\|\cdot\|_{D}\right)$ be two Banach spaces with $D$ densely embedded in $X$, and denote by $\mathcal{D}$ an open subset of $D$. We consider the abstract initial value problem

$$
u^{\prime}(t)=F(u(t)), \quad t>0, \quad u(0) \in \mathcal{D} .
$$

Derivatives with respect to the argument of a function are henceforth denoted by a prime. Our assumptions on the nonlinearity $F$ are the following.

Assumption 2.1. We assume that the function $F: \mathcal{D} \rightarrow X$ is Fréchet differentiable and that its derivative $F^{\prime}: \mathcal{D} \rightarrow L(D, X)$ has the following properties.

(i) $F^{\prime}$ is locally Lipschitz continuous; i.e., for each $u^{*} \in \mathcal{D}$ there exist $R>0$ and $L>0$ such that

$$
\left\|F^{\prime}(v)-F^{\prime}(w)\right\|_{D \rightarrow X} \leq L\|v-w\|_{D}
$$

for all $v, w \in \mathcal{D}$ with $\left\|v-u^{*}\right\|_{D} \leq R$ and $\left\|w-u^{*}\right\|_{D} \leq R$.

(ii) For every $u^{*} \in \mathcal{D}$ the operator $F^{\prime}\left(u^{*}\right)$ is sectorial; i.e., there exist $\theta \in(0, \pi / 2)$, $\omega_{0} \in \mathbb{R}$ and $M>0$ such that if $z \in \mathbb{C}$ and $\left|\arg \left(z-\omega_{0}\right)\right| \leq \pi-\theta$, then $z-F^{\prime}\left(u^{*}\right)$ has a bounded inverse in $X$ and

$$
\left\|\left(z-F^{\prime}\left(u^{*}\right)\right)^{-1}\right\|_{X \rightarrow X} \leq \frac{M}{\left|z-\omega_{0}\right|} .
$$

(iii) For every $u^{*} \in \mathcal{D}$ the graph-norm of $F^{\prime}\left(u^{*}\right)$ is equivalent to the norm of $D$.

Under these assumptions, it is known that (2.1) has a locally unique solution (see [12. Theorem 8.1.1]). This solution $u \in C([0, \delta], D) \cap C^{1}([0, \delta], X)$ has the regularity property $u \in C_{\alpha}^{\alpha}((0, \delta], D)$ for arbitrary $0<\alpha<1$. For the convenience of the reader we recall the definition of the space $C_{\alpha}^{\alpha}$. For a Banach space $\left(B,\|\cdot\|_{B}\right)$, $C_{\alpha}^{\alpha}((0, \delta], B)$ is the space of all bounded functions $v:(0, \delta] \rightarrow B$ such that $t \mapsto t^{\alpha} v(t)$

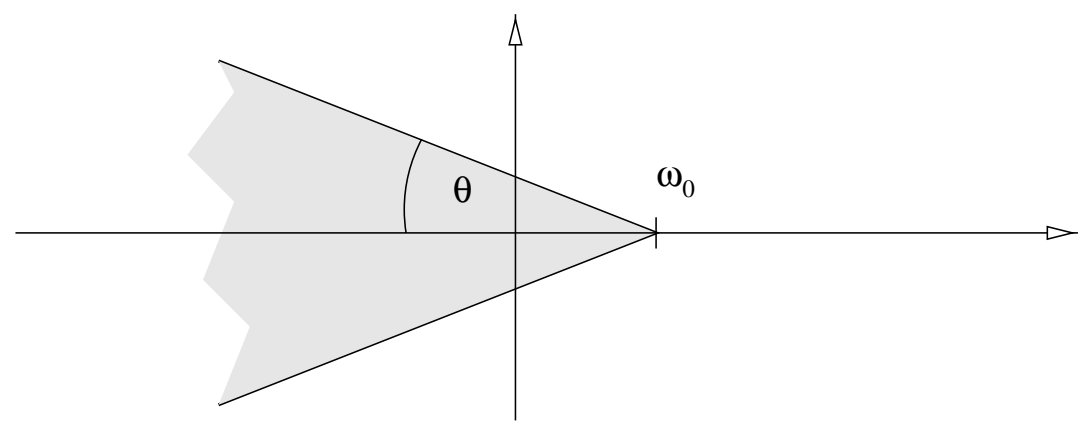

Figure 1. Condition (2.31) holds for all $z$ outside the shaded cone. 
is $\alpha$-Hölder continuous in $(0, \delta]$. This space is endowed with the norm

$$
\|v\|_{C_{\alpha}^{\alpha}((0, \delta], B)}=\sup _{0<t \leq \delta}\|v(t)\|_{B}+\sup _{0<s<t \leq \delta} \frac{\|v(t)-v(s)\|_{B}}{(t-s)^{\alpha}} s^{\alpha} .
$$

We next give two nonlinear initial-boundary value problems that fit into our framework. More examples can be found in [12].

Example 2.2 (Combustion of a solid fuel, [3] Section 6.7]). Let $U(t, x)$ denote the temperature of a combusting solid fuel at position $x \in[0,1]$ and time $t \geq 0$. A model for the evolution of $U$ is given by the nonlinear initial-boundary value problem

$$
\partial_{t} U(t, x)=\partial_{x}\left(k\left(\partial_{x} U(t, x)\right) \partial_{x} U(t, x)\right)+\varphi(U(t, x)), \quad 0<x<1, \quad t>0,
$$

with homogeneous Neumann boundary conditions $\partial_{x} U(t, 0)=\partial_{x} U(t, 1)=0$ for all $t>0$ and initial condition $U(0, x)=U_{0}(x)$ for $0<x<1$. We assume that the diffusion coefficient $k$ is twice differentiable, with bounded second derivative, and that it satisfies the uniform ellipticity condition

$$
k(y)+y k^{\prime}(y) \geq \kappa>0 \quad \text { for all } y \in \mathbb{R} .
$$

We further suppose that $\varphi$ has a locally Lipschitz continuous derivative and that the initial value $U_{0}$ is twice continuously differentiable and satisfies the compatibility conditions $U_{0}^{\prime}(0)=U_{0}^{\prime}(1)=0$.

Choosing $X=C([0,1])$ and $D=\left\{v \in C^{2}([0,1]): v^{\prime}(0)=v^{\prime}(1)=0\right\}$ allows us to write (2.4) in the abstract form (2.1) with $u(t)=U(t, \cdot)$ and

$$
F(v)=\left(k\left(v^{\prime}\right) v^{\prime}\right)^{\prime}+\varphi(v) .
$$

The smoothness assumptions on $k$ and $\varphi$ immediately imply condition (i) of Assumption 2.1, and the ellipticity condition (2.5) implies (ii) and (iii) there.

Equally, it can be shown that our assumptions are satisfied for the Banach spaces $X=L^{2}(0,1)$ and $D=\left\{v \in H^{2}(0,1): v^{\prime}(0)=v^{\prime}(1)=0\right\}$. This follows from the well-known embedding $H^{1}(0,1) \subset C([0,1])$.

Example 2.3 (Semilinear problem with moving boundary). We consider the semilinear parabolic problem

$$
\partial_{t} V(t, y)=\partial_{y y} V(t, y)+\varphi\left(V(t, y), \partial_{y} V(t, y)\right), \quad 0<y<b(t), \quad t>0,
$$

with homogeneous Dirichlet boundary conditions $V(t, 0)=V(t, b(t))=0$ for $t>0$ and initial condition $V(0, y)=V_{0}(y)$ for $0<y<b(t)$. Here the position of the right boundary $b(t)$ is determined by the ordinary differential equation

$$
\partial_{t} b(t)=\psi\left(b(t), V(t, b(t)), \partial_{y} V(t, b(t))\right), \quad t>0, \quad b(0)=1 .
$$

We assume that $\varphi$ and $\psi$ have locally Lipschitz continuous derivatives and that $V_{0}$ is twice continuously differentiable with $V_{0}(0)=V_{0}(1)=0$.

The famous Stefan problem that models the melting of ice is of this form by taking $\varphi=0$ and $\psi(p, q, r)=-\beta r$ with a positive constant $\beta$ (see [16, Section 15.4]). 
Changing the variables $U(t, x)=V(t, b(t) x)$ transforms problem (2.6) to the interval $0 \leq x \leq 1$, and we obtain the nonlinear system

$$
\begin{aligned}
& \partial_{t} U(t, x)=\frac{\partial_{x x} U(t, x)}{b(t)^{2}}+\varphi\left(U(t, x), \frac{\partial_{x} U(t, x)}{b(t)}\right)+\frac{x \partial_{t} b(t)}{b(t)} \partial_{x} U(t, x), \\
& \partial_{t} b(t)=\psi\left(b(t), U(t, 1), \frac{\partial_{x} U(t, 1)}{b(t)}\right), \quad 0<x<1, \quad t>0
\end{aligned}
$$

with boundary conditions $U(t, 0)=U(t, 1)=0$ for $t>0$ and initial conditions $U(0, x)=V_{0}(x)$ for $0<x<1$ and $b(0)=1$.

We choose $X=C([0,1]) \times \mathbb{R}$ and $D=\left\{v \in C^{2}([0,1]): v(0)=v(1)=0\right\} \times \mathbb{R}$, and since the projection $P: C([0,1]) \rightarrow \mathbb{R}: v \mapsto v(1)$ is continuous, the conditions (i), (ii), and (iii) of Assumption 2.1 are again easily verified.

We finish this section by introducing some notation. The aim of the paper is the analysis of backward Euler discretizations of (2.1) which are given as sequences $u_{0}, u_{1}, \ldots, u_{N}$ in $\mathcal{D}$, corresponding to a grid $0=t_{0}<t_{1}<\cdots<t_{N} \leq T$. This motivates the consideration of the following discrete norms and seminorms in $X^{N}$ :

$$
\begin{gathered}
\mu(\mathbf{v})=\sup _{1 \leq n \leq N}\left\|v_{n}\right\|, \quad \lambda_{\alpha}(\mathbf{v})=\sup _{1 \leq k<n \leq N} \frac{\left\|v_{n}-v_{k}\right\|}{\left(t_{n}-t_{k}\right)^{\alpha}} t_{k}^{\alpha}, \\
\|\mathbf{v}\|_{\alpha}=\mu(\mathbf{v})+\lambda_{\alpha}(\mathbf{v}),
\end{gathered}
$$

for $\mathbf{v}=\left(v_{n}\right)_{n=1}^{N} \in X^{N}$ and $0<\alpha<1$. Analogously, we denote

$$
\begin{gathered}
\mu_{D}(\mathbf{v})=\sup _{1 \leq n \leq N}\left\|v_{n}\right\|_{D}, \quad \lambda_{D, \alpha}(\mathbf{v})=\sup _{\substack{1 \leq k<n \leq N \\
1 \leq k}} \frac{\left\|v_{n}-v_{k}\right\|_{D}}{\left(t_{n}-t_{k}\right)^{\alpha}} t_{k}^{\alpha}, \\
\|\mathbf{v}\|_{D, \alpha}=\mu_{D}(\mathbf{v})+\lambda_{D, \alpha}(\mathbf{v}),
\end{gathered}
$$

for $\mathbf{v} \in D^{N}$. Further we define $\mu_{\beta}$ for $0 \leq \beta \leq 1$ and $\mathbf{v} \in X_{\beta}^{N}$ through

$$
\mu_{\beta}(\mathbf{v})=\sup _{1 \leq n \leq N}\left\|v_{n}\right\|_{\beta}
$$

Here $\left(X_{\beta},\|\cdot\|_{\beta}\right)$ denotes the real interpolation space $(X, D)_{\beta, \infty}$ between $X$ and $D$ (see [12, Section 1.2.1]). Note that \|\|$\cdot\|\|_{\alpha}$ and $\|\mid \cdot\| \|_{D, \alpha}$ are discrete versions of the norms $\|\cdot\|_{C_{\alpha}^{\alpha}((0, \delta], X)}$ and $\|\cdot\|_{C_{\alpha}^{\alpha}((0, \delta], D)}$, respectively.

\section{Convergence analysis of the BaCKWARd Euler solution}

In this section we study the backward Euler method for discretizing (2.1) in time. We show that a unique numerical solution exists for finite times, provided that the maximal stepsize is chosen sufficiently small. We further derive convergence estimates under various smoothness assumptions on the exact solution.

We first consider a local situation for which more precise estimates can be obtained. For this, it is convenient to linearize (2.1) around the initial value $u(0)$. This gives the (formally) semilinear problem

$$
u^{\prime}=A u+f(u), \quad t>0, \quad u(0) \in \mathcal{D},
$$

where $A=F^{\prime}(u(0))$ and $f(u)=F(u)-A u$ for $u \in \mathcal{D}$. In view of (2.2), there exist $R>0$ and $L>0$ such that

$$
\|f(v)-f(w)\| \leq L \varrho\|v-w\|_{D}
$$

for all $\|v-u(0)\|_{D} \leq \varrho \leq R$ and $\|w-u(0)\|_{D} \leq \varrho \leq R$ (see proof of Lemma 5.2). 
Since the backward Euler method is invariant under linearization, the numerical approximation $u_{n}$ to $u\left(t_{n}\right)$ is given by the recursion

$$
\frac{u_{n}-u_{n-1}}{h_{n}}=A u_{n}+f\left(u_{n}\right), \quad 1 \leq n \leq N,
$$

with $t_{n}=t_{n-1}+h_{n}$ for $1 \leq n \leq N$ and $t_{0}=0$. Here $h_{n}>0$ denotes the stepsize which is chosen according to accuracy requirements. The starting value $u_{0} \in \mathcal{D}$ is allowed to be different from $u(0)$.

We remark that, due to (2.3) with $u^{*}=u(0)$ and (3.2), the nonlinear equation (3.3) has a unique solution $u_{n} \in \mathcal{D}$ for stepsizes $h_{n}$ satisfying $h_{n} \omega_{0}<1$, as long as $\left\|u_{n-1}-u(0)\right\|_{D} \leq \varrho$ for a certain $\varrho>0$. In fact, (3.3) can be solved by standard fixed-point iteration (see Lemma 5.2). Let us point out, however, that already after one single step we can only expect

$$
\left\|u_{1}-u(0)\right\|_{D} \leq C \varrho,
$$

where $C>1$. Thus, after a finite number of steps, independently of the stepsizes, the validity of (3.2) is no longer guaranteed. Therefore, this step-by-step approach is not suited to construct the numerical solution on a finite time interval $[0, T]$.

In order to overcome this difficulty, we adopt a global approach relying on the discrete variation-of-constants formula

$$
u_{n}=r\left(t_{n}, 0\right) u_{0}+\sum_{k=1}^{n} h_{k} r\left(t_{n}, t_{k-1}\right) f\left(u_{k}\right),
$$

where the discrete transition operator $r\left(t_{n}, t_{k}\right)$ is defined by

$$
r\left(t_{n}, t_{k}\right)=\left(1-h_{n} A\right)^{-1} \cdots\left(1-h_{k+1} A\right)^{-1}, \quad 0 \leq k<n \leq N,
$$

and $r\left(t_{k}, t_{k}\right)=1$. Note that this operator is well defined for

$$
\max _{1 \leq k \leq N} h_{k} \leq \bar{h}, \quad \text { if } \quad \bar{h} \omega_{0}<1 .
$$

The numerical solution of (3.1) can be constructed by fixed-point iteration in (3.4). This is based on the fact that the nonlinear operator

$$
\Phi: \mathcal{B} \subset \mathcal{D}^{N} \longrightarrow \mathcal{D}^{N}: \mathbf{v} \longmapsto \Phi(\mathbf{v})=\mathbf{r} u_{0}+\mathcal{K}(f(\mathbf{v})),
$$

with $\mathbf{r}=\left(r\left(t_{n}, 0\right)\right)_{n=1}^{N}, f(\mathbf{v})=\left(f\left(v_{n}\right)\right)_{n=1}^{N}$ for $\mathbf{v}=\left(v_{n}\right)_{n=1}^{N} \in \mathcal{D}^{N}$, and

$$
\mathcal{K}(\mathbf{w})=\left(\sum_{k=1}^{n} h_{k} r\left(t_{n}, t_{k-1}\right) w_{k}\right)_{n=1}^{N} \quad \text { for } \mathbf{w}=\left(w_{n}\right)_{n=1}^{N} \in X^{N},
$$

is a contraction for a suitably chosen subset $\mathcal{B}$. Unfortunately, it turns out that the interval of existence is limited by the fact that $\left\|\mid \Phi\left(\mathbf{u}_{0}\right)-\mathbf{u}_{0}\right\| \|_{D, \alpha}$ has to be sufficiently small, for $\mathbf{u}_{0}=\left(u_{0}, \ldots, u_{0}\right)^{N}$. Thus, nothing can be said about the size of $t_{N}$ in this approach. This kind of difficulty also appears when constructing the continuous solution (see [12, Theorem 8.1.1]).

However, the global approach based on the convolution operator in (3.7b) turns out to be useful in order to derive preliminary convergence estimates. Eventually, these estimates can be used to establish the existence of the numerical solution for finite times.

Assume for a moment that the backward Euler approximations $u_{0}, u_{1}, \ldots, u_{N}$ to the solution exist. We set $\widetilde{u}_{n}=u\left(t_{n}\right)$ and denote the errors by $e_{n}=\widetilde{u}_{n}-u_{n}$. 
Inserting the exact solution into the numerical scheme defines the defects $d_{n}$ by

$$
\frac{\widetilde{u}_{n}-\widetilde{u}_{n-1}}{h_{n}}=A \widetilde{u}_{n}+f\left(\widetilde{u}_{n}\right)+d_{n}, \quad 1 \leq n \leq N .
$$

Subtracting (3.3) from this identity gives the error recursion

$$
\mathbf{e}=\mathbf{r} e_{0}+\mathcal{K}(f(\widetilde{\mathbf{u}})-f(\mathbf{u}))+\mathcal{K}(\mathbf{d}),
$$

where $\mathbf{e}=\left(e_{1}, e_{2}, \ldots, e_{N}\right)^{T} \in \mathcal{D}^{N}$, etc.

Let $C_{3}$ and $R$ be the constants provided by Lemma 5.3 for $u^{*}=u(0)$. We will show below that after a possible reduction of $T$, we may assume that there exists $0<\varrho \leq R$ such that

$$
\begin{gathered}
\mu_{D}(\widetilde{\mathbf{u}}-\mathbf{u}(0)) \leq \varrho, \quad \mu_{D}(\mathbf{u}-\mathbf{u}(0)) \leq \varrho, \\
C_{3} C_{5}\left(2 \varrho+\lambda_{D, \alpha}(\widetilde{\mathbf{u}})\right) \leq \gamma<1
\end{gathered}
$$

where $0<\alpha<1$ is chosen and $C_{5}$ is the constant appearing in Lemma 5.5. Taking norms in (3.8) and using Lemmas 5.35 .4 and 5.5 yields

$$
\|\mathbf{e} \mid\|_{D, \alpha} \leq \frac{1}{1-\gamma}\left(C_{4}\left\|e_{0}\right\|_{D}+\|\| \mathcal{K}(\mathbf{d})\|\|_{D, \alpha}\right) .
$$

Depending on our requirements on the analytical solution, we obtain different bounds for $\left|\|\mathcal{K}(\mathbf{d}) \mid\|_{D, \alpha}\right.$ and consequently different error estimates (see Theorems 3.1 and 3.2 below). We finally point out that because of

$$
\left\|e_{n}\right\|_{D} \leq\|\mathbf{e}\|_{D, \alpha}
$$

these theorems also provide error estimates in $D$.

Theorem 3.1. Let $u:[0, T] \rightarrow D$ be a solution of 2.1) with $u^{\prime \prime} \in C_{\alpha}^{\alpha}((0, T], X)$ and assume that

$$
C_{3} C_{5}\left(2\|u-u(0)\|_{L^{\infty}([0, T], D)}+\|u\|_{C_{\alpha}^{\alpha}((0, T], D)}\right)<1,
$$

where $C_{3}$ and $C_{5}$ are the constants provided by Lemmas 5.3 and 5.5 for $u^{*}=u(0)$. Suppose that either

(a) the stepsizes $h_{n}=h$ are constant, or

(b) the stepsizes verify $h_{n} \geq \sigma h_{n-1}, 2 \leq n \leq N$, for some $\sigma>0$.

Then there exist constants $h^{*}>0, \varrho_{0}>0$ and $C>0$ such that the backward Euler solution $u_{n}$ exists for stepsizes satisfying $0<h_{n} \leq h^{*}$ and for initial values $u_{0}$ with $\left\|u_{0}-u(0)\right\|_{D} \leq \varrho_{0}$, as long as $t_{n} \leq T$. Further, we have the error bounds

$$
\|\mathbf{e}\|_{D, \alpha} \leq C\left(\left\|e_{0}\right\|_{D}+h\left\|u^{\prime \prime}\right\|_{C_{\alpha}^{\alpha}((0, T], X)}\right)
$$

for constant stepsizes, and

$$
\|\mathbf{e} \mid\|_{D, \alpha} \leq C\left(\left\|e_{0}\right\|_{D}+\max _{1 \leq m \leq n \leq N}\left(\left(h_{n}^{1-\alpha}+h_{m+1}^{1-\alpha}\right) M_{n}+h_{m}^{1-\alpha} M_{m, n}\right)\right)
$$

with

$$
M_{n}=\left\|u^{\prime \prime}\right\|_{L^{\infty}\left(\left[t_{n-1}, t_{n}\right], X\right)}, \quad M_{m, n}=\sup _{t_{m-1}<s<t \leq t_{n}} \frac{\left\|u^{\prime \prime}(t)-u^{\prime \prime}(s)\right\|}{(t-s)^{\alpha}} s^{\alpha}
$$

for variable stepsizes, respectively. The constant $C$ depends on $\alpha, T$ and on $C_{5}$ of Lemma 5.5. For variable stepsizes, it further depends on $\sigma$. 
Proof. Set $\varrho_{1}=\|u-u(0)\|_{L^{\infty}([0, T], D)}$ and choose $\varrho_{1}<\varrho \leq R$ such that

$$
C_{3} C_{5}\left(2 \varrho+\|u\|_{C_{\alpha}^{\alpha}((0, T], D)}\right)<1 .
$$

In the first part of the proof we show the validity of the error estimates (3.12) and (3.13) under the assumptions that the numerical solution $\left(u_{n}\right)_{n=1}^{N}$ is defined as long as $t_{N} \leq T$ and that

$$
\left\|u_{n}-u(0)\right\|_{D} \leq \varrho, \quad n \leq N .
$$

In the second part we justify these assumptions.

(i) In view of (3.10) and Lemma 5.5, we have to estimate $\|\mathbf{d}\| \|_{\alpha}$. Taylor series expansion shows that the defects are given by

$$
d_{n}=h_{n} \int_{0}^{1} \tau u^{\prime \prime}\left(t_{n}-\tau h_{n}\right) \mathrm{d} \tau .
$$

This immediately yields

$$
\mu(\mathbf{d}) \leq 1 / 2 \max _{1 \leq n \leq N} h_{n} M_{n} .
$$

For estimating $\lambda_{D, \alpha}(\mathbf{d})$ we first write for $m<n$

$$
\begin{gathered}
d_{n}-d_{m}=h_{m} \int_{0}^{1} \tau\left(u^{\prime \prime}\left(t_{n}-\tau h_{n}\right)-u^{\prime \prime}\left(t_{m}-\tau h_{m}\right)\right) \mathrm{d} \tau \\
+\left(h_{n}-h_{m}\right) \int_{0}^{1} \tau u^{\prime \prime}\left(t_{n}-\tau h_{n}\right) \mathrm{d} \tau .
\end{gathered}
$$

For constant stepsizes the second term in (3.15) drops and the estimate

$$
\frac{\left\|d_{n}-d_{m}\right\|}{\left(t_{n}-t_{m}\right)^{\alpha}} t_{m}^{\alpha} \leq h M_{m, n} \int_{0}^{1} \tau\left(\frac{m}{m-\tau}\right)^{\alpha} \mathrm{d} \tau \leq \frac{M_{m, n}}{1-\alpha} h
$$

proves the first part of the theorem.

For variable stepsizes, one has

$$
\begin{gathered}
\frac{\left\|d_{n}-d_{m}\right\|}{\left(t_{n}-t_{m}\right)^{\alpha}} t_{m}^{\alpha} \leq h_{m} M_{m, n}\left(\frac{t_{n}-t_{m}+h_{m}}{t_{n}-t_{m}}\right)^{\alpha} \int_{0}^{1} \tau\left(\frac{t_{m}}{t_{m}-\tau h_{m}}\right)^{\alpha} \mathrm{d} \tau \\
+\frac{\left|h_{n}-h_{m}\right|}{\left(t_{n}-t_{m}\right)^{\alpha}} t_{m}^{\alpha} \frac{M_{n}}{2} .
\end{gathered}
$$

Due to our assumptions on the stepsize sequence, we have

$$
\left(\frac{t_{n}-t_{m}+h_{m}}{t_{n}-t_{m}}\right)^{\alpha} \leq\left(1+\frac{1}{\sigma}\right)^{\alpha}
$$

and

$$
\frac{\left|h_{n}-h_{m}\right|}{\left(t_{n}-t_{m}\right)^{\alpha}} t_{m}^{\alpha} \leq\left(h_{n}^{1-\alpha}+\frac{1}{\sigma} h_{m}^{1-\alpha}\right) T^{\alpha} .
$$

The remaining term in (3.16) is bounded as follows:

$$
h_{m} \int_{0}^{1} \tau\left(\frac{t_{m}}{t_{m}-\tau h_{m}}\right)^{\alpha} \mathrm{d} \tau \leq h_{m}^{1-\alpha} T^{\alpha} \int_{0}^{1} \tau\left(\frac{h_{m}}{t_{m}-\tau h_{m}}\right)^{\alpha} \mathrm{d} \tau \leq \frac{h_{m}^{1-\alpha} T^{\alpha}}{1-\alpha} .
$$

Inserting these bounds into (3.16) gives the required bound for $\lambda_{\alpha}(\mathbf{d})$.

(ii) It remains to show that the backward Euler solution exists and that (3.14) holds. The idea of the proof is simple and standard for nonlinear equations: as long as $u_{n-1}$ remains sufficiently close to $\widetilde{u}_{n-1}$, (3.3) can be solved for $u_{n}$ and the above error estimate ensures that $u_{n}$ is close enough to $\widetilde{u}_{n}$ as well. Repeating this 
process proves the desired result. However, we have to pay some attention to the parameters involved.

For simplicity, we give the proof for constant stepsizes only. Set $\varrho_{2}=\varrho-\varrho_{1}$, and let $0<\varrho_{*}<\varrho^{*} \leq \varrho_{2}$ and $h^{*} \leq \underline{\mathrm{h}}$ denote the thresholds provided by Lemma 5.2 , applied to $\varrho_{2}$ and $u^{*}$, where $u^{*}$ varies in the compact set formed by the values $u(t)$, $0 \leq t \leq T$. After a possible reduction of $h^{*}$, we can choose $\varrho_{0}>0$ such that

$$
C\left(\varrho_{0}+h^{*}\left\|u^{\prime \prime}\right\|_{C_{\alpha}^{\alpha}((0, T], X)}\right) \leq \varrho_{*} / 2,
$$

where $C$ is the constant from (3.12). Since $u$ is uniformly continuous, we can further assume that $\left\|u\left(t_{n}\right)-u\left(t_{n-1}\right)\right\|_{D} \leq \varrho_{*} / 2$ for $h \leq h^{*}$.

Suppose by induction that $u_{n}$ exists and that (3.14) is satisfied for $n \leq m$. Then, due to (3.12) and the above choice of parameters, the bound

$$
\left\|e_{m}\right\|_{D} \leq C\left(\left\|e_{0}\right\|_{D}+h\left\|u^{\prime \prime}\right\|_{C_{\alpha}^{\alpha}((0, T], X)}\right) \leq \varrho_{*} / 2 \quad \text { for } h \leq h^{*}
$$

implies

$$
\left\|u_{m}-\widetilde{u}_{m+1}\right\|_{D} \leq\left\|e_{m}\right\|_{D}+\left\|\widetilde{u}_{m}-\widetilde{u}_{m+1}\right\|_{D} \leq \varrho_{*} \cdot
$$

An application of Lemma 5.2 with $u^{*}=\widetilde{u}_{m+1}$ and $w=u_{m}$ shows that $u_{m+1}$ exists and $\left\|e_{m+1}\right\|_{D} \leq \varrho^{*}$. Consequently, the estimate

$$
\left\|u_{m+1}-u(0)\right\|_{D} \leq\left\|e_{m+1}\right\|_{D}+\left\|\widetilde{u}_{m+1}-u(0)\right\|_{D} \leq \varrho_{2}+\left(\varrho-\varrho_{2}\right)=\varrho
$$

follows. This yields (3.14) and concludes the proof.

In practice it might be difficult to know whether $u^{\prime \prime}$ belongs to $C_{\alpha}^{\alpha}((0, T], X)$. This limitation is overcome in the next theorem where we impose the natural condition

$$
A u(0)+f(u(0)) \in X_{\beta},
$$

for some $\alpha<\beta \leq 1$. Note that, in actual applications, $X_{\beta}$ is often a Sobolev space that does not depend on the boundary conditions for $\beta$ sufficiently small. Hence, if the initial value is sufficiently smooth, this condition is easily seen to be satisfied.

It is also known that under (3.17) the exact solution of (2.1) has the additional regularity properties $u^{\prime} \in L^{\infty}\left([0, T], X_{\beta}\right) \cap C^{\beta}([0, T], X)$ (see [12, Theorem 8.1.3]).

Theorem 3.2. Let $u:[0, T] \rightarrow \mathcal{D}$ be a solution of (2.1) such that (3.11) is satisfied and assume that (3.17) holds for some $0<\alpha<\beta \leq 1$.

Then there exist constants $h^{*}>0, \varrho_{0}>0$ and $C>0$ such that, for arbitrary stepsizes $0<h_{n} \leq h^{*}$ and for initial values $u_{0}$ with $\left\|u_{0}-u(0)\right\|_{D} \leq \varrho_{0}$, the backward Euler solution $u_{n}$ is defined as long as $t_{n} \leq T$ and we have

$$
\|\mathbf{e}\|_{D, \alpha} \leq C\left(\left\|e_{0}\right\|_{D}+\max _{1 \leq n \leq N} h_{n}^{\beta-\alpha} I_{n}^{\alpha / \beta} J_{n}^{1-\alpha / \beta}\right)
$$

with

$$
I_{n}=\left\|u^{\prime}\right\|_{L^{\infty}\left(\left[t_{n-1}, t_{n}\right], X_{\beta}\right)}, \quad J_{n}=\left\|u^{\prime}\right\|_{C^{\beta}\left(\left[t_{n-1}, t_{n}\right], X\right)} .
$$

The constant $C$ depends on $\alpha, \beta, T$ and on $C_{6}$ of Lemma 5.6 .

Proof. We follow the arguments of the proof of Theorem 3.1 Therefore, it is sufficient to establish the validity of (3.18) under the assumptions that the numerical solution $\left(u_{n}\right)_{n=1}^{N}$ exists as long as $t_{N} \leq T$ and that (3.9) holds for some $\varrho \leq R$. 
In view of (3.10) and Lemma 5.6, we have to estimate $\mu_{\alpha}(\mathbf{d})$. For the defects $d_{n}$, we use the representation

$$
d_{n}=\int_{0}^{1}\left(u^{\prime}\left(t_{n}-\tau h_{n}\right)-u^{\prime}\left(t_{n}\right)\right) \mathrm{d} \tau
$$

to obtain the estimates

$$
\left\|d_{n}\right\| \leq \frac{h_{n}^{\beta}}{1+\beta} J_{n} \leq h_{n}^{\beta} J_{n} \quad \text { and } \quad\left\|d_{n}\right\|_{\beta} \leq 2 I_{n} .
$$

By a standard interpolation argument

$$
\left\|d_{n}\right\|_{\alpha} \leq\left\|d_{n}\right\|^{1-\alpha / \beta}\left\|d_{n}\right\|_{\beta}^{\alpha / \beta}
$$

we get

$$
\mu_{\alpha}(\mathbf{d}) \leq 2 \max _{1 \leq n \leq N} h^{\beta-\alpha} I_{n}^{\alpha / \beta} J_{n}^{1-\alpha / \beta},
$$

which yields the desired result.

The previous theorems are local in nature. By applying them recursively, we obtain pointwise convergence estimates in $D$ for finite times. In the following theorem, the number $\alpha$ has the same meaning as in the local results before.

Theorem 3.3. Let $u:[0, T] \rightarrow \mathcal{D}$ be a solution of (2.1) and let $0<\alpha<1$. Assume that either

(a) $u^{\prime \prime} \in C_{\alpha}^{\alpha}((0, T], X)$ and the stepsizes $h_{n}=h$ are constant, or

(b) $u^{\prime \prime} \in C_{\alpha}^{\alpha}((0, T], X)$ and $h_{n} \geq \sigma h_{n-1}, 2 \leq n \leq N$, for some $\sigma>0$, or

(c) $A u(0)+f(u(0)) \in X_{\beta}$ for some $0<\alpha<\beta \leq 1$.

Then there exist constants $h^{*}>0, \delta>0$ and $C>0$ such that the backward Euler solution $u_{n}$ exists for stepsizes satisfying $0<h_{n} \leq h^{*}$ and for initial values $u_{0}$ with $\left\|u_{0}-u(0)\right\|_{D} \leq \delta$, as long as $t_{n} \leq T$. For $0 \leq n \leq N$, we have the error bounds

(a) $\left\|e_{n}\right\|_{D} \leq C\left(\left\|e_{0}\right\|_{D}+h\left\|u^{\prime \prime}\right\|_{C_{\alpha}^{\alpha}\left(\left(0, t_{N}\right], X\right)}\right)$, or

(b) $\left\|e_{n}\right\|_{D} \leq C\left(\left\|e_{0}\right\|_{D}+\max _{1 \leq m \leq k \leq N}\left(\left(h_{k}^{1-\alpha}+h_{m+1}^{1-\alpha}\right) M_{k}+h_{m}^{1-\alpha} M_{m, k}\right)\right)$, or

(c) $\left\|e_{n}\right\|_{D} \leq C\left(\left\|e_{0}\right\|_{D}+\max _{1 \leq m \leq N} h_{m}^{\beta-\alpha} I_{m}^{\alpha / \beta} J_{m}^{1-\alpha / \beta}\right)$,

respectively.

Proof. We only give the proof of the first result. The remaining statements follow in a similar way.

Since $u$ is continuous, there are constants $R>0$ and $L>0$ such that (2.2) is uniformly satisfied for $u^{*}$ varying in the set formed by the values $u(t), 0 \leq t \leq T$. Moreover, by Lemma [5.8, there exists a partition $0=T_{0}<T_{1}<\cdots<T_{J}=T$ of $[0, T]$ such that

$$
C_{3} C_{5}\left(2\left\|u_{T_{j}}-u\left(T_{j-1}\right)\right\|_{L^{\infty}\left(\left[0, H_{j}\right], D\right)}+\left\|u_{T_{j}}\right\|_{C_{\alpha}^{\alpha}\left(\left(0, H_{j}\right], D\right)}\right)<1, \quad 1 \leq j \leq J,
$$

with $H_{j}=T_{j}-T_{j-1}$ and $u_{T_{j}}(t)=u\left(T_{j-1}+t\right), 0 \leq t \leq H_{j}$. Here $C_{3}$ and $C_{5}$ are the constants provided by Lemmas 5.3 and $\left[5.5\right.$ for $u^{*}=u\left(T_{j}\right)$ and $R$. Notice that these constants only depend on $R$ and $L$. 
Therefore, we deduce from Theorem 3.1 applied piece-by-piece, that there exist positive constants $h^{*}$ and $\widetilde{C}$, such that for $0<h \leq h^{*}$

$$
\left\|e_{n}\right\|_{D} \leq C^{j}\left\|e_{0}\right\|_{D}+\widetilde{C} h\left\|u^{\prime \prime}\right\|_{C_{\alpha}^{\alpha}\left(\left(0, T_{j}\right], X\right)}, \quad 0 \leq t_{n} \leq T_{j} .
$$

After a possible reduction of $\delta$ and $h^{*}$, this estimate shows that $\left\|e_{n}\right\|_{D} \leq \varrho_{0}$, with $\varrho_{0}$ given by Theorem 3.1. Notice that $J$ is independent of $0<h \leq h^{*}$. The desired error estimate thus follows by recursion.

\section{BehaViour NeAR AN ASYMPtotically STABle EQUILIBRiUm}

In this section we study the long-term behaviour of time discretizations of (2.1). To keep our exposition in a reasonable length, we restrict our attention to hyperbolic equilibria. For these the principle of linearized stability holds, which means that the dynamical behaviour near such an equilibrium $\bar{u}$ is fully determined by the linearized equation

$$
v^{\prime}=F^{\prime}(\bar{u})(v-\bar{u})
$$

(see [12, Section 9.1]). We show that a similar property holds for the backward Euler discretization of (2.1). Further, numerical simulations that illustrate our theoretical result are given.

For notational simplicity, we concentrate on the asymptotically stable case. Let $\bar{u} \in \mathcal{D}$ be an equilibrium of (2.1), i.e., $F(\bar{u})=0$, and assume that the sectorial operator

$$
A=F^{\prime}(\bar{u}) \quad \text { is asymptotically stable, i.e., } \omega_{0}<0 .
$$

The number $\omega_{0}$ is defined in (2.3) (see also Figure 1). In this situation, it is well known that $\bar{u}$ is asymptotically stable and attracts all solutions in a sufficiently small neighbourhood of $\bar{u}$ with exponential speed. More precisely, it is shown in 12, Theorem 9.1.2] that for each $\omega<\left|\omega_{0}\right|$ there are constants $\delta_{0}>0$ and $C>0$ such that the solution of (2.1) exists for all positive times and satisfies

$$
\|u(t)-\bar{u}\|_{D} \leq C \cdot \mathrm{e}^{-\omega t}\|u(0)-\bar{u}\|_{D}, \quad \text { for all } t \geq 0,
$$

whenever the initial value satisfies $\|u(0)-\bar{u}\|_{D} \leq \delta_{0}$.

The following theorem gives the corresponding result for the backward Euler discretization. Note that any equilibrium of (2.1) is also an equilibrium of the backward Euler discretization.

Theorem 4.1. Let $\bar{u}$ be an equilibrium of (2.1) and assume that (4.1) holds. Then, for any choice of $\omega<\left|\omega_{0}\right|$, there are positive constants $\bar{h}, \delta$ and $C$ such that the following holds. The backward Euler solution $\left(u_{n}\right)_{n=1}^{\infty}$ of (2.1) exists for all stepsize sequences satisfying $0<h_{n} \leq \bar{h}$ and for all initial values $u_{0}$ with $\left\|u_{0}-\bar{u}\right\|_{D} \leq \delta$, and we have

$$
\left\|u_{n}-\bar{u}\right\|_{D} \leq C \cdot \mathrm{e}^{-\omega t_{n}}\left\|u_{0}-\bar{u}\right\|_{D}, \quad \text { for all } n \geq 0 .
$$

Note that the constant $C$ depends on $\omega$, but not on the particular choice of the stepsize sequence.

Demanding that the numerical solution decays towards the equilibrium nearly as fast as the exact solution imposes a severe restriction on the maximal stepsize. This restriction is overcome in the following theorem, where exponentially fast convergence is obtained, if the stepsizes remain bounded. 
Theorem 4.2. Let $\bar{u}$ be an equilibrium of (2.1) and assume that (4.1) holds. Then, for any $\bar{h}>0$, there are constants $0<\omega<\left|\omega_{0}\right|, \delta>0$ and $C>0$ such that the backward Euler solution $\left(u_{n}\right)_{n=1}^{\infty}$ of (2.1) exists for all stepsize sequences satisfying $0<h_{n} \leq \bar{h}$ and for all initial values $u_{0}$ with $\left\|u_{0}-\bar{u}\right\|_{D} \leq \delta$, and (4.3) holds.

Proof of Theorem 4.1. We linearize (2.1) around the equilibrium $\bar{u}$ and construct the backward Euler solution by fixed-point iteration. In order to capture the decaying behaviour of the solution we use exponentially weighted norms. For $\omega>0$ and sequences $\mathbf{v}=\left(v_{n}\right)_{n=1}^{\infty}$ in $D$, we modify (2.8b) in the following way:

$$
\begin{gathered}
\mu_{D, \omega}(\mathbf{v})=\sup _{1 \leq n<\infty} \mathrm{e}^{\omega t_{n}}\left\|v_{n}\right\|_{D}, \quad \lambda_{D, \alpha, \omega}(\mathbf{v})=\sup _{1 \leq k<n<\infty} \mathrm{e}^{\omega t_{k}} \frac{\left\|v_{n}-v_{k}\right\|_{D}}{\left(t_{n}-t_{k}\right)^{\alpha}} t_{k}^{\alpha}, \\
\|\mathbf{v}\|_{D, \alpha, \omega}=\mu_{D, \omega}(\mathbf{v})+\lambda_{D, \alpha, \omega}(\mathbf{v}),
\end{gathered}
$$

as well as the corresponding norm $\|\cdot\|_{\alpha, \omega}$ based on $\|\cdot\|$. A crucial observation is that Lemmas 5.3 5.4 and 5.5 have an extension to these exponentially weighted norms for $0<\omega<\left|\omega_{1}\right|<\left|\omega_{0}\right|$ with $\omega_{1}$ as in Lemma 5.1. The gap $\omega-\left|\omega_{1}\right|$ is needed to bound the powers of $t_{n}$ that are encountered.

With these preparations, we are ready to give the proof. Let $\mathcal{B}$ denote the ball

$$
\mathcal{B}=\left\{\mathbf{v} \in \mathcal{D}^{\infty}:\|\mathbf{v}-\overline{\mathbf{u}}\|_{D, \alpha, \omega} \leq \varrho\right\} .
$$

We define $\Phi$ as in (3.7a) with $N=\infty$. Using the above-mentioned extensions of Lemmas 5.3 and 5.5 with $u^{*}=\bar{u}$ proves

$$
\left\|\left|\Phi(\mathbf{v})-\Phi(\mathbf{w})\|\|_{D, \alpha, \omega} \leq 3 \varrho C_{3} C_{5}\right|\right\| \mathbf{v}-\mathbf{w} \|_{D, \alpha, \omega}
$$

for $\mathbf{v}, \mathbf{w} \in \mathcal{B}$. This shows that $\Phi$ is a contraction on $\mathcal{B}$ with contraction factor $1 / 2$ for $\varrho$ sufficiently small.

It remains to show that $\Phi$ maps $\mathcal{B}$ onto $\mathcal{B}$ if $u_{0}$ lies sufficiently close to $\bar{u}$. Since

$$
\Phi(\overline{\mathbf{u}})=\mathbf{r} u_{0}+(1-\mathbf{r}) \bar{u},
$$

we have for all $\mathbf{v} \in \mathcal{B}$

$$
\begin{aligned}
\|\Phi(\mathbf{v})-\overline{\mathbf{u}}\|_{D, \alpha, \omega} & \leq\|\Phi(\mathbf{v})-\Phi(\overline{\mathbf{u}})\|_{D, \alpha, \omega}+\|\Phi(\overline{\mathbf{u}})-\overline{\mathbf{u}}\|_{D, \alpha, \omega} \\
& \leq 1 / 2\|\mathbf{v}-\overline{\mathbf{u}}\|_{D, \alpha, \omega}+\left\|\mathbf{r}\left(u_{0}-\bar{u}\right)\right\|_{D, \alpha, \omega}
\end{aligned}
$$

The last term is estimated by the first part of Lemma 5.4

$$
\left\|\mathbf{r}\left(u_{0}-\bar{u}\right)\right\|\left\|_{D, \alpha, \omega} \leq C_{4}\right\| u_{0}-\bar{u} \|_{D} .
$$

For $\delta$ satisfying $2 \delta C_{4} \leq \varrho$, we thus have $\Phi(\mathcal{B}) \subset \mathcal{B}$.

This proves the existence of a unique fixed-point $\mathbf{u}$, which is the searched backward Euler solution. Using further

$$
\left\|\left|\Phi(\mathbf{u})-\overline{\mathbf{u}}\left\|\left.\right|_{D, \alpha, \omega} \leq 1 / 2|\|\Phi(\mathbf{u})-\overline{\mathbf{u}}\||_{D, \alpha, \omega}+\right\| \mathbf{r}\left(u_{0}-\bar{u}\right)\right|\right\|_{D, \alpha, \omega}
$$

yields

$$
\|\mathbf{u}-\overline{\mathbf{u}}\|_{D, \alpha, \omega} \leq 2 C_{4}\left\|u_{0}-\bar{u}\right\|_{D} .
$$

In particular, we get

$$
\sup _{1 \leq n<\infty} \mathrm{e}^{\omega t_{n}}\left\|u_{n}-\bar{u}\right\|_{D} \leq 2 C_{4}\left\|u_{0}-\bar{u}\right\|_{D}
$$

which proves the assertion of the theorem.

Proof of Theorem 4.2. The proof is very similar to the preceding one and therefore omitted. It relies on the stability bounds given in Lemma 5.1, part (b). 
TABle 1. Numerically observed contraction factors

\begin{tabular}{|r|c|c|c|c|c|c|}
\hline$h$ & 1.0000 & 0.5000 & 0.2500 & 0.1250 & 0.0625 & 0.03125 \\
\hline$\omega_{h}$ & 0.4055 & 0.4463 & 0.4711 & 0.4849 & 0.4922 & 0.4960 \\
\hline
\end{tabular}

We close this section with a numerical example that illustrates Theorem 4.1

Example 4.3 (Combustion of a solid fuel). We take up Example 2.2 and specify the functions $k, \varphi$ and $U_{0}$ as follows:

$$
k(y)=y^{2}+1, \quad \varphi(y)=-y(y-1 / 2)(y-1), \quad U_{0}(x)=1 / 2+2 x^{2}(1-x)^{2} .
$$

Note that the initial condition $U_{0}$ is compatible with the boundary conditions and that $k$ satisfies the ellipticity condition (2.5) with $\kappa=1$. The problem has three equlibria $\bar{u}=1, \bar{u}=0$, and $\bar{u}=1 / 2$. The first two are asymptotically stable with $\omega_{0}=-1 / 2$. Due to our choice of $U_{0}$, we expect convergence to $\bar{u}=1$.

The partial differential equation (2.4) is discretized in space by standard finite differences on an equidistant grid with meshwidth $1 / 200$ and in time by the backward Euler method with constant stepsize $h$. For different values of $h$, the integration is performed up to $t=40$. The numerical approximations $\omega_{h}$ to $\omega$ are displayed in Table 1. The results are in complete agreement with Theorem 4.1 .

\section{LEMMAS}

In this section we collect the auxiliary results that are needed in the proofs of the previous theorems. Throughout the section we set

$$
f(u)=F(u)-A u, \quad \text { where } A=F^{\prime}\left(u^{*}\right)
$$

for some $u^{*} \in \mathcal{D}$ and denote by $\omega_{0} \in \mathbb{R}$ the constant from (2.3) that corresponds to $u^{*}$. We fix $\kappa>\omega_{0}$ and $\bar{h}>0$ such that $\bar{h} \omega_{0}<1$ and consider arbitrary grid points $0=t_{0}<t_{1}<\cdots<t_{N}$ that satisfy $h_{n}=t_{n}-t_{n-1} \leq \bar{h}$. There is no restriction on the maximal stepsize for $\omega_{0} \leq 0$.

For the discrete transition operators (3.5), we have the following stability bounds.

Lemma 5.1. (a) For any $\omega_{1}>\omega_{0}$ there exist constants $h^{*}>0$ and $C_{1}>0$ such that for $0 \leq \nu \leq 1$

$$
\left\|(\kappa-A)^{\nu} r\left(t_{n}, t_{k}\right)\right\|_{X \rightarrow X} \leq C_{1} \frac{\mathrm{e}^{\omega_{1}\left(t_{n}-t_{k}\right)}}{\left(t_{n}-t_{k}\right)^{\nu}}, \quad 0 \leq k<n,
$$

whenever the stepsizes are bounded by $h^{*}$.

(b) Let $\omega_{0}<0$ and $h^{*}>0$. Then there exist constants $\omega_{0}<\omega_{1}<0$ and $C_{1}>0$ such that (5.2) holds, whenever the stepsizes are bounded by $h^{*}$.

Similar bounds are given in 2, 4, 14. We note for later use that (5.2) also holds for $1 \leq \nu \leq 2$ if $k<n-1$.

Proof. The estimate (5.2) is a consequence of the stability bounds

$$
\left\|\mathrm{e}^{t A}\right\|_{X \rightarrow X} \leq C \mathrm{e}^{\omega_{0} t}, \quad\left\|A \mathrm{e}^{t A}\right\|_{X \rightarrow X} \leq \frac{C}{t} \mathrm{e}^{\omega_{0} t}, \quad t>0,
$$

for the analytic semigroup. Using the representation

$$
r\left(t_{n}, t_{k}\right)=\int_{0}^{\infty} \cdots \int_{0}^{\infty} \mathrm{e}^{-s_{k+1}-\ldots-s_{n}} \mathrm{e}^{\left(s_{k+1} h_{k+1}+\ldots+s_{n} h_{n}\right) A} \mathrm{~d} s_{k+1} \cdots \mathrm{d} s_{n}
$$


shows

$$
\begin{gathered}
\left\|r\left(t_{n}, t_{k}\right)\right\|_{X \rightarrow X} \leq C \prod_{j=k+1}^{n}\left(1-h_{j} \omega_{0}\right)^{-1}, \\
\left\|A r\left(t_{n}, t_{k}\right)\right\|_{X \rightarrow X} \leq C \int_{-\infty}^{\omega_{0}} \prod_{j=k+1}^{n}\left(1-h_{j} \omega\right)^{-1} \mathrm{~d} \omega .
\end{gathered}
$$

For $\omega \leq \omega_{0} \leq 0$, we have

$$
1-h_{j} \omega \geq\left(1-h_{j} \omega_{0}\right)\left(1+c h_{j}\left(\omega_{0}-\omega\right)\right)
$$

with $c=1-h^{*} \omega_{0}$. Arguing as in [4] shows

$$
\left\|A r\left(t_{n}, t_{k}\right)\right\|_{X \rightarrow X} \leq C \frac{1-h^{*} \omega_{0}}{t_{n}-t_{k}} \prod_{j=k+1}^{n}\left(1-h_{j} \omega_{0}\right)^{-1} .
$$

For $\omega_{0}>0$ we use an idea from [6] and eliminate the small steps by

$$
\left(1-h_{j} \omega\right)\left(1-h_{k} \omega\right) \geq 1-\left(h_{j}+h_{k}\right) \omega,
$$

until (5.3) is again satisfied. Part (a) of the lemma then follows from standard estimates and interpolation.

In order to verify (b) we note that the function

$$
\omega_{1}=\omega_{1}(H)=-\frac{\log \left(1-H \omega_{0}\right)}{H}
$$

is monotonically increasing for $\omega_{0}<0$ with $\omega_{1}(0)=\omega_{0}$ and $\omega_{1}(\infty)=0$. Hence,

$$
\left(1-h_{j} \omega_{0}\right)^{-1} \leq \mathrm{e}^{\omega_{1} h_{j}}
$$

with $\omega_{1}$ given by (5.4) for $H=\max h_{j} \leq h^{*}$.

We note for later use that the identity

$$
r\left(t_{n}, t_{k}\right)-1=\sum_{l=k+1}^{n} h_{l} A r\left(t_{n}, t_{l-1}\right)
$$

together with Lemma 5.1 implies for $0 \leq k<n \leq N$ and $0<\nu \leq 1$ the bound

$$
\left\|\left(r\left(t_{n}, t_{k}\right)-1\right)(\kappa-A)^{-\nu}\right\|_{X \rightarrow X} \leq \frac{C_{1}}{\nu} \mathrm{e}^{\omega_{1}^{+}\left(t_{n}-t_{k}\right)}\left(t_{n}-t_{k}\right)^{\nu}
$$

with $\omega_{1}^{+}=\max \left(\omega_{1}, 0\right)$. For simplicity, we make no notational difference between the constants in (5.2) and (5.5).

Lemma 5.2. Let $u^{*} \in \mathcal{D}$ and $R_{0}>0$. Then there exist $0<\varrho_{*}<\varrho^{*} \leq R_{0}$ and $h^{*}>0$ such that, for $w \in \mathcal{D}$ with $\left\|w-u^{*}\right\|_{D} \leq \varrho_{*}$ and for $0<h \leq h^{*}$, the equation

$$
\frac{v-w}{h}=A v+f(v)
$$

possesses a unique solution $v \in \mathcal{D}$ with $\left\|v-u^{*}\right\|_{D} \leq \varrho^{*}$. Moreover, the quantities $\varrho_{*}, \varrho^{*}$ and $h^{*}$ can be chosen uniformly for $u^{*}$ belonging to a relatively compact subset of $\mathcal{D}$. 
Proof. We first note that, due to (2.2), there exist $R>0$ and $L>0$ such that

$$
\left\|f^{\prime}(v)-f^{\prime}(w)\right\|_{D \rightarrow X} \leq L\|v-w\|_{D},
$$

for all $v, w \in \mathcal{D}$ with $\left\|v-u^{*}\right\|_{D} \leq R$ and $\left\|w-u^{*}\right\|_{D} \leq R$. Therefore, since $f^{\prime}\left(u^{*}\right)=0$, we also have

$$
\left\|f^{\prime}(v)\right\|_{D \rightarrow X} \leq L \varrho
$$

for all $v \in \mathcal{D}$ such that $\left\|v-u^{*}\right\|_{D} \leq \varrho \leq R$. This implies that

$$
\|f(v)-f(w)\| \leq L \varrho\|v-w\|_{D},
$$

for all $v, w \in \mathcal{D}$ with $\left\|v-u^{*}\right\|_{D} \leq \varrho \leq R$ and $\left\|w-u^{*}\right\|_{D} \leq \varrho \leq R$.

Equation (5.6) is equivalent to $v=g(v)$, where $g$ is defined by

$$
g(v)=(1-h A)^{-1} w+h(1-h A)^{-1} f(v) .
$$

We solve (5.6) by fixed-point iteration in the set $\mathcal{B}=\left\{v \in \mathcal{D}:\left\|v-u^{*}\right\|_{D} \leq \varrho^{*}\right\}$. For this, we have to show that $g$ is contractive and maps $\mathcal{B}$ onto $\mathcal{B}$, for $\varrho_{*}$ and $\varrho^{*}$ sufficiently small.

By the equivalence of $\|\cdot\|_{D}$ with the graph-norm of $A$, there exists $\bar{M}$ such that

$$
\left\|h(1-h A)^{-1}\right\|_{X \rightarrow D} \leq \bar{M}, \quad\left\|(1-h A)^{-1}\right\|_{D \rightarrow D} \leq \bar{M} \quad \text { for } 0<h \leq \bar{h} .
$$

On the one hand, we have

$$
\|g(v)-g(\widetilde{v})\|_{D} \leq \bar{M} L \varrho^{*}\|v-\widetilde{v}\|_{D},
$$

for $h \leq \bar{h}$ and $v, \widetilde{v} \in \mathcal{D}$ with $\left\|v-u^{*}\right\|_{D} \leq \varrho^{*}$ and $\left\|\widetilde{v}-u^{*}\right\|_{D} \leq \varrho^{*}$. On the other hand, it holds

$$
\begin{aligned}
g(v)-u^{*}= & (1-h A)^{-1}\left(w-u^{*}\right)+h(1-h A)^{-1}\left(A u^{*}+f\left(u^{*}\right)\right) \\
& +h(1-h A)^{-1}\left(f(v)-f\left(u^{*}\right)\right)
\end{aligned}
$$

so that

$$
\left\|g(v)-u^{*}\right\|_{D} \leq \bar{M}\left\|w-u^{*}\right\|_{D}+\left\|h(1-h A)^{-1}\left(A u^{*}+f\left(u^{*}\right)\right)\right\|+\bar{M} L \varrho^{*}\left\|v-u^{*}\right\|_{D} .
$$

In view of these bounds, if we choose $\varrho_{*}$ and $\varrho^{*}$ such that $\bar{M} \varrho_{*} \leq \varrho^{*} / 3$ and $\bar{M} L \varrho^{*} \leq$ $1 / 3$, then $g$ is a contraction on $\mathcal{B}$. Moreover, since $h(1-h A)^{-1} \rightarrow 0$ strongly as an operator from $X$ to $D$, we can select $h^{*} \leq \bar{h}$ such that, for $0<h \leq h^{*}$,

$$
\left\|h(1-h A)^{-1}\left(A u^{*}+f\left(u^{*}\right)\right)\right\|_{D} \leq \varrho^{*} / 3 .
$$

Thus, $g$ maps $\mathcal{B}$ into $\mathcal{B}$, and the fixed-point theorem provides the existence of a unique solution $v$ of (5.6).

Since $F^{\prime}$ is locally Lipschitz continuous, $R_{0}$ and $L$ can be taken uniformly for $u^{*}$ in a compact set. Moreover, the equivalence of $\|\cdot\|_{D}$ with the graph-norm of $F^{\prime}\left(u^{*}\right)$ is also uniform on the compact set. With this, the statement of the lemma follows easily.

Lemma 5.3. For $0<\alpha<1$ there exist constants $C_{3}>0$ and $R>0$ such that

$$
\|f(\mathbf{v})-f(\mathbf{w})\|_{\alpha} \leq C_{3}\left(2 \varrho+\lambda_{D, \alpha}(\mathbf{w})\right)\|\mathbf{v}-\mathbf{w}\|_{D, \alpha}
$$

for all $\mathbf{v}$ and $\mathbf{w}$ in the set $\mathcal{V}=\left\{\widetilde{\mathbf{v}} \in \mathcal{D}^{N}: \mu_{D}\left(\widetilde{\mathbf{v}}-\mathbf{u}^{*}\right) \leq \varrho\right\}$ whenever $0<\varrho \leq R$. 
Proof. Choose $R$ as in (2.2) and let $\mathbf{v}, \mathbf{w} \in \mathcal{V}$ for some $0<\varrho \leq R$. In view of (5.9), we have

$$
\mu(f(\mathbf{v})-f(\mathbf{w})) \leq L \varrho \mu_{D}(\mathbf{v}-\mathbf{w}),
$$

and thus it remains to bound $\lambda_{\alpha}(f(\mathbf{v})-f(\mathbf{w}))$. We set $G_{n}=f^{\prime}\left(\sigma v_{n}+(1-\sigma) w_{n}\right)$, $0 \leq \sigma \leq 1$, and write for $m<n$

$$
\begin{aligned}
\left(f\left(v_{n}\right)\right. & \left.-f\left(w_{n}\right)\right)-\left(f\left(v_{m}\right)-f\left(w_{m}\right)\right)=\int_{0}^{1}\left(G_{n}\left(v_{n}-w_{n}\right)-G_{m}\left(v_{m}-w_{m}\right)\right) \mathrm{d} \sigma \\
& =\int_{0}^{1} G_{n}\left(\left(v_{n}-w_{n}\right)-\left(v_{m}-w_{m}\right)\right) \mathrm{d} \sigma+\int_{0}^{1}\left(G_{n}-G_{m}\right)\left(v_{m}-w_{m}\right) \mathrm{d} \sigma
\end{aligned}
$$

Using (5.8), we can estimate the first term on the right-hand side by

$$
\int_{0}^{1}\left\|G_{n}\left(\left(v_{n}-w_{n}\right)-\left(v_{m}-w_{m}\right)\right)\right\| \mathrm{d} \sigma \leq L \varrho \lambda_{D, \alpha}(\mathbf{v}-\mathbf{w})\left(t_{n}-t_{m}\right)^{\alpha} t_{m}^{-\alpha} .
$$

Due to (5.7), the remaining term can be bounded as follows:

$$
\begin{aligned}
\int_{0}^{1} & \left\|\left(G_{n}-G_{m}\right)\left(v_{m}-w_{m}\right)\right\| \mathrm{d} \sigma \\
& \leq L \int_{0}^{1}\left(\left\|w_{n}-w_{m}\right\|_{D}+\sigma\left\|\left(v_{n}-w_{n}\right)-\left(v_{m}-w_{m}\right)\right\|_{D}\right) \mathrm{d} \sigma\left\|v_{m}-w_{m}\right\|_{D} \\
& \leq L\left(\lambda_{D, \alpha}(\mathbf{w})+1 / 2 \lambda_{D, \alpha}(\mathbf{v}-\mathbf{w})\right) \mu_{D}(\mathbf{v}-\mathbf{w})\left(t_{n}-t_{m}\right)^{\alpha} t_{m}^{-\alpha} .
\end{aligned}
$$

The above estimates readily give

$$
\lambda_{\alpha}(f(\mathbf{v})-f(\mathbf{w})) \leq L\left(2 \varrho \lambda_{D, \alpha}(\mathbf{v}-\mathbf{w})+\lambda_{D, \alpha}(\mathbf{w}) \mu_{D}(\mathbf{v}-\mathbf{w})\right),
$$

and this inequality combined with (5.11) proves (5.10).

In Lemmas 5.4, 5.5 and 5.6 below we establish certain estimates involving $\|\cdot\|_{D, \alpha}$. As $\|\cdot\|_{D}$ is equivalent to the graph-norm of $A$, the norm

$$
\|\mathbf{v}\|_{\alpha}+\|A \mathbf{v} \mid\|_{\alpha}, \quad \mathbf{v} \in D^{N}
$$

is equivalent to \|\|$\cdot\|\|_{D, \alpha}$ as well, for all $0<\alpha<1$. Since the required estimates for \|\|$\cdot \|_{\alpha}$ are usually obtained more easily (and in a similar way) than the corresponding estimates for $\|A \cdot\|_{\alpha}$, we give for simplicity the proofs only for $\|A \cdot\|_{\alpha}$. Henceforth, $C$ denotes a generic constant that possibly depends on $C_{1}$ and on constants that arise from changing between equivalent norms.

Lemma 5.4. Let $0<\alpha<1$.

(a) There exists a constant $C_{4}>0$ such that for every $v \in D$

$$
\|\mathbf{r} v\|_{D, \alpha} \leq C_{4}\|v\|_{D} .
$$

The constant $C_{4}$ depends on $t_{N}$, but it is otherwise independent of the grid. If $\omega_{0}$ is nonnegative, then $C_{4}$ is bounded for finite times. If $\omega_{0}$ is negative, then $C_{4}$ can be chosen independently of $t_{N}$.

(b) For $x \in X$ we have

$$
\lim _{t_{N} \rightarrow 0}\|(\mathbf{r}-1) x\|_{\alpha}=0 .
$$

The convergence is uniform on relatively compact subsets of $X$. 
Proof. In order to prove the first statement of the lemma, we have to estimate $A\left(r\left(t_{n}, 0\right)-r\left(t_{m}, 0\right)\right) v$. Using the identity

$$
r\left(t_{n}, 0\right)-r\left(t_{m}, 0\right)=\left(r\left(t_{n}, t_{m}\right)-1\right) r\left(t_{m}, 0\right)
$$

we obtain

$$
\begin{aligned}
\left\|A\left(r\left(t_{n}, 0\right)-r\left(t_{m}, 0\right)\right) v\right\| \leq C \|\left(r\left(t_{n}, t_{m}\right)\right. & -1)(\kappa-A)^{-\alpha} \|_{X \rightarrow X} \\
& \times\left\|(\kappa-A)^{\alpha} r\left(t_{m}, 0\right)\right\|_{X \rightarrow X}\|v\|_{D} .
\end{aligned}
$$

With the help of (5.2) and (5.5) the right-hand side can be bounded by

$$
\frac{C}{\alpha} \mathrm{e}^{\omega_{1}^{+} t_{N}}\|v\|_{D}\left(t_{n}-t_{m}\right)^{\alpha} t_{m}^{-\alpha}
$$

which proves the first result.

To show the second statement of the lemma we choose $\widetilde{x} \in D$. From the identity

$$
r\left(t_{k}, 0\right)-r\left(t_{k-1}, 0\right)=h_{k} A r\left(t_{k}, 0\right)
$$

we get

$$
\begin{aligned}
\|\left(r\left(t_{n}, 0\right)\right. & \left.-r\left(t_{m}, 0\right)\right) x \| \leq \sum_{k=m+1}^{n} h_{k}\left(\left\|A r\left(t_{k}, 0\right)(x-\widetilde{x})\right\|+\left\|r\left(t_{k}, 0\right) A \widetilde{x}\right\|\right) \\
& \leq C t_{m}^{-\alpha} \sum_{k=m+1}^{n} h_{k} t_{k}^{-1+\alpha} \mathrm{e}^{\omega_{1} t_{k}}\|x-\widetilde{x}\|+C \sum_{k=m+1}^{n} h_{k} \mathrm{e}^{\omega_{1} t_{k}}\|\widetilde{x}\|_{D} \\
& \leq C\left(\|x-\widetilde{x}\|+t_{N}\|\widetilde{x}\|_{D}\right) \mathrm{e}^{\omega_{1}^{+} t_{N}}\left(t_{n}-t_{m}\right)^{\alpha} t_{m}^{-\alpha} .
\end{aligned}
$$

Since $D$ is dense in $X$, the second statement of the lemma follows.

Lemma 5.5. For $0<\alpha<1$ there exists a constant $C_{5}>0$ such that if $\|\mathbf{v}\| \|_{\alpha}<\infty$ we have

$$
\|\mathcal{K}(\mathbf{v}) \mid\|_{D, \alpha} \leq C_{5}\|\mathbf{v}\|_{\alpha} .
$$

The constant $C_{5}$ depends on $t_{N}$, but it is otherwise independent of the grid. If $\omega_{0}$ is nonnegative, then $C_{5}$ is bounded for finite times. If $\omega_{0}$ is negative, then $C_{5}$ can be chosen independently of $t_{N}$.

Proof. Analogously to the modified variation-of-constants formula (1.3) and with the help of

$$
h_{k} A r\left(t_{n}, t_{k-1}\right)=r\left(t_{n}, t_{k-1}\right)-r\left(t_{n}, t_{k}\right),
$$

we split $\mathcal{K}(\mathbf{v})$ such that $A \mathcal{K}(\mathbf{v})=\mathbf{a}+\mathbf{b}$ where

$$
\begin{gathered}
a_{n}=\sum_{k=1}^{n} h_{k} A r\left(t_{n}, t_{k-1}\right)\left(v_{k}-v_{n}\right), \\
b_{n}=\sum_{k=1}^{n} h_{k} A r\left(t_{n}, t_{k-1}\right) v_{n}=\left(r\left(t_{n}, 0\right)-1\right) v_{n} .
\end{gathered}
$$

According to this we have to estimate the four terms $\mu(\mathbf{a}), \lambda_{\alpha}(\mathbf{a}), \mu(\mathbf{b})$, and $\lambda_{\alpha}(\mathbf{b})$. 
(i) Using (5.2) we get

$$
\begin{aligned}
\left\|a_{n}\right\| & \leq C_{1} \lambda_{\alpha}(\mathbf{v}) \sum_{k=1}^{n} h_{k} \frac{\left(t_{n}-t_{k}\right)^{\alpha}}{\left(t_{n}-t_{k-1}\right) t_{k}^{\alpha}} \mathrm{e}^{\omega_{1}\left(t_{n}-t_{k-1}\right)} \\
& \leq C B(\alpha, 1-\alpha) \mathrm{e}^{\omega_{1}^{+} t_{N}} \lambda_{\alpha}(\mathbf{v}),
\end{aligned}
$$

where $B$ denotes the beta function. The last bound follows from comparing with the integral in a similar way as in Lemma 5.7.

(ii) In order to estimate $\lambda_{\alpha}(\mathbf{a})$ we use the identity

$$
\begin{aligned}
a_{n}-a_{m}= & \sum_{k=m+1}^{n} h_{k} A r\left(t_{n}, t_{k-1}\right)\left(v_{k}-v_{n}\right)+\sum_{k=1}^{m} h_{k} A r\left(t_{n}, t_{k-1}\right)\left(v_{m}-v_{n}\right) \\
& +\sum_{k=1}^{m} h_{k} A\left(r\left(t_{n}, t_{k-1}\right)-r\left(t_{m}, t_{k-1}\right)\right)\left(v_{k}-v_{m}\right) \\
= & S_{1}+S_{2}+S_{3} .
\end{aligned}
$$

We take norms and use again (5.2) and (5.5). This gives

$$
\begin{aligned}
\left\|S_{1}\right\| & \leq C_{1} \mathrm{e}^{\omega_{1}^{+} t_{N}} \lambda_{\alpha}(\mathbf{v}) \sum_{k=m+1}^{n} \frac{h_{k}}{\left(t_{n}-t_{k-1}\right)^{1-\alpha} t_{k}^{\alpha}} \\
& \leq \alpha^{-1} C_{1} \mathrm{e}^{\omega_{1}^{+} t_{N}} \lambda_{\alpha}(\mathbf{v})\left(t_{n}-t_{m}\right)^{\alpha} t_{m}^{-\alpha},
\end{aligned}
$$

and, together with (5.12),

$$
\begin{aligned}
\left\|S_{2}\right\| & \leq\left\|r\left(t_{n}, t_{m}\right)\left(r\left(t_{m}, 0\right)-1\right)\left(v_{m}-v_{n}\right)\right\| \\
& \leq C_{1}\left(1+C_{1}\right) \mathrm{e}^{\omega_{1}^{+} t_{N}} \lambda_{\alpha}(\mathbf{v})\left(t_{n}-t_{m}\right)^{\alpha} t_{m}^{-\alpha} .
\end{aligned}
$$

With the help of Lemma 5.7 we get

$$
\begin{aligned}
\left\|S_{3}\right\| & \leq \sum_{k=1}^{m} h_{k} \sum_{l=m+1}^{n} h_{l}\left\|A^{2} r\left(t_{l}, t_{k-1}\right)\left(v_{k}-v_{m}\right)\right\| \\
& \leq C_{1} \lambda_{\alpha}(\mathbf{v}) \sum_{k=1}^{m} h_{k} \sum_{l=m+1}^{n} h_{l} \mathrm{e}^{\omega_{1}\left(t_{l}-t_{k-1}\right)} \frac{\left(t_{m}-t_{k}\right)^{\alpha}}{\left(t_{l}-t_{k-1}\right)^{2} t_{k}^{\alpha}} \\
& \leq \frac{4 C_{1}}{\alpha(1-\alpha)} \mathrm{e}^{\omega_{1}^{+} t_{N}} \lambda_{\alpha}(\mathbf{v})\left(t_{n}-t_{m}\right)^{\alpha} t_{m}^{-\alpha}
\end{aligned}
$$

which proves the estimate for $\lambda_{\alpha}(\mathbf{a})$.

(iii) The stability bound (5.2) for the transition operator immediately gives

$$
\mu(\mathbf{b}) \leq\left(1+C_{1} \mathrm{e}^{\omega_{1}^{+} t_{N}}\right) \mu(\mathbf{v}) .
$$

(iv) For the estimate of $\lambda_{\alpha}(\mathbf{b})$ we write

$$
\begin{aligned}
b_{n}-b_{m}= & \left(r\left(t_{n}, 0\right)-1\right) v_{n}-\left(r\left(t_{m}, 0\right)-1\right) v_{m} \\
= & \left(r\left(t_{n}, t_{m}\right)-1\right)(\kappa-A)^{-\alpha}(\kappa-A)^{\alpha} r\left(t_{m}, 0\right) v_{n} \\
& +\left(r\left(t_{m}, 0\right)-1\right)\left(v_{n}-v_{m}\right) .
\end{aligned}
$$

A further application of (5.2) and (5.5) yields

$$
\left\|b_{n}-b_{m}\right\| \leq\left(\frac{C_{1}^{2}}{\alpha} \mathrm{e}^{\omega_{1}^{+} t_{N}} \mu(\mathbf{v})+\left(1+C_{1} \mathrm{e}^{\omega_{1}^{+} t_{N}}\right) \lambda_{\alpha}(\mathbf{v})\right)\left(t_{n}-t_{m}\right)^{\alpha} t_{m}^{-\alpha} .
$$


This finally concludes the proof of the lemma.

The following lemma is used in the proof of Theorem 3.2. For the definition of the norm $\mu_{\alpha}$, we refer to (2.9).

Lemma 5.6. For $0<\alpha<1$ there exists a constant $C_{6}>0$ such that

$$
\|\mathcal{K}(\mathbf{v})\|_{D, \alpha} \leq C_{6} \mu_{\alpha}(\mathbf{v}) \quad \text { for } \mathbf{v} \in \mathcal{D}^{N} .
$$

The constant $C_{6}$ depends on $t_{N}$, but it is otherwise independent of the grid.

Proof. Using (5.2) we have for $\mathbf{w}=\mathcal{K}(\mathbf{v})$

$$
\begin{aligned}
\left\|w_{n}\right\|_{D} & \leq C \sum_{k=1}^{n} h_{k}\left\|(\kappa-A)^{1-\alpha} r\left(t_{n}, t_{k-1}\right)\right\|_{X \rightarrow X}\left\|(\kappa-A)^{\alpha} v_{k}\right\| \\
& \leq C \mathrm{e}^{\omega_{1}^{+} t_{N}} \mu_{\alpha}(\mathbf{v}) \sum_{k=1}^{n} \frac{h_{k}}{\left(t_{n}-t_{k-1}\right)^{1-\alpha}}
\end{aligned}
$$

and further, by comparing the sum with the corresponding integral,

$$
\mu_{D}(\mathbf{w}) \leq \frac{C t_{N}^{\alpha}}{\alpha} \mathrm{e}^{\omega_{1}^{+} t_{N}} \mu_{\alpha}(\mathbf{v}) .
$$

In order to estimate $\lambda_{D, \alpha}(\mathbf{w})$, we split $A\left(w_{n}-w_{m}\right)=S_{1}+S_{2}$ where

$$
S_{1}=\sum_{k=m+1}^{n} h_{k} A r\left(t_{n}, t_{k-1}\right) v_{k}
$$

and, due to $r\left(t_{l}, t_{k-1}\right)-r\left(t_{l-1}, t_{k-1}\right)=h_{l} A r\left(t_{l}, t_{k}\right)$, we get

$$
S_{2}=\sum_{k=1}^{m} h_{k} A\left(r\left(t_{n}, t_{k-1}\right)-r\left(t_{m}, t_{k-1}\right)\right) v_{k}=\sum_{k=1}^{m} h_{k} \sum_{l=m+1}^{n} h_{l} A^{2} r\left(t_{l}, t_{k-1}\right) v_{k} .
$$

As before, we premultiply $v_{k}$ with $(\kappa-A)^{\alpha}$ and use (5.2) and the corresponding integrals to estimate $S_{1}$ and $S_{2}$ by

$$
\begin{aligned}
& \left\|S_{1}\right\| \leq \frac{C t_{N}^{\alpha}}{\alpha} \mathrm{e}^{\omega_{1}^{+} t_{N}} \mu_{\alpha}(\mathbf{v})\left(t_{n}-t_{m}\right)^{\alpha} t_{m}^{-\alpha}, \\
& \left\|S_{2}\right\| \leq \frac{C t_{N}^{\alpha}}{\alpha(1-\alpha)} \mathrm{e}^{\omega_{1}^{+} t_{N}} \mu_{\alpha}(\mathbf{v})\left(t_{n}-t_{m}\right)^{\alpha} t_{m}^{-\alpha} .
\end{aligned}
$$

This concludes the proof of the lemma.

Lemma 5.7. The inequality

$$
\sum_{k=1}^{m} h_{k} \sum_{l=m+1}^{n} h_{l} \frac{\left(t_{m}-t_{k}\right)^{\alpha}}{\left(t_{l}-t_{k-1}\right)^{2} t_{k}^{\alpha}} \leq \frac{4}{\alpha(1-\alpha)}\left(t_{n}-t_{m}\right)^{\alpha} t_{m}^{-\alpha}
$$

holds for $1 \leq m<n \leq N$.

Proof. By comparing with the corresponding integral, we get

$$
\sum_{l=m+1}^{n} \frac{h_{l}}{\left(t_{l}-t_{k-1}\right)^{2}} \leq \frac{t_{n}-t_{m}}{\left(t_{m}-t_{k-1}\right)\left(t_{n}-t_{k-1}\right)} \quad \text { for } 1 \leq k \leq m .
$$

We thus have to estimate

$$
\left(t_{n}-t_{m}\right) \sum_{k=1}^{m} \frac{h_{k}}{\left(t_{m}-t_{k-1}\right)^{1-\alpha} t_{k}^{\alpha}\left(t_{n}-t_{k-1}\right)} .
$$


For this we consider the function

$$
G(s)=\frac{1}{\left(t_{m}-s\right)^{1-\alpha} s^{\alpha}\left(t_{n}-s\right)}, \quad 0<s<t_{m} .
$$

Let $t^{*}$ be the point where $G$ attains its minimum, and let $1 \leq p \leq m$ be the index such that $t_{p-1} \leq t^{*} \leq t_{p}$. We split the sum into three parts (from 1 to $p-1$, the term with $k=p$, and from $p$ to $m$ ) and compare each part with a corresponding integral. By means of the variable change $\sigma t_{m}=s$, we get

$$
\left(t_{n}-t_{m}\right) \sum_{k=1}^{m} \frac{h_{k}}{\left(t_{m}-t_{k-1}\right)^{1-\alpha} t_{k}^{\alpha}\left(t_{n}-t_{k-1}\right)} \leq \theta \int_{0}^{1} \frac{\mathrm{d} \sigma}{(1-\sigma)^{1-\alpha} \sigma^{\alpha}(\theta+1-\sigma)},
$$

where $\theta=\left(t_{n}-t_{m}\right) / t_{m}$. The elementary estimates

$$
\theta \int_{0}^{1 / 2} \frac{\mathrm{d} \sigma}{(1-\sigma)^{1-\alpha} \sigma^{\alpha}(\theta+1-\sigma)} \leq \frac{2 \theta}{2 \theta+1} \frac{1}{1-\alpha} \leq \frac{2 \theta^{\alpha}}{1-\alpha}
$$

and

$$
\theta \int_{1 / 2}^{1} \frac{\mathrm{d} \sigma}{(1-\sigma)^{1-\alpha} \sigma^{\alpha}(\theta+1-\sigma)} \leq 2^{\alpha} \theta^{\alpha} \int_{0}^{\infty} \frac{\mathrm{d} \tau}{(1+\tau) \tau^{1-\alpha}} \leq \frac{\theta^{\alpha}}{\alpha}+\frac{2 \theta^{\alpha}}{1-\alpha},
$$

where we used $1-\sigma=\theta \tau$, finally prove the lemma.

Lemma 5.8. Let $u:[0, T] \rightarrow \mathcal{D}$ be a solution of (2.1). Then, for every $\sigma>0$, there exists a partition $0=T_{0}<T_{1} \cdots<T_{J}=T$ such that

$$
2\left\|u_{T_{j}}-u\left(T_{j-1}\right)\right\|_{L^{\infty}\left(\left[0, H_{j}\right], D\right)}+\left\|u_{T_{j}}\right\|_{C_{\alpha}^{\alpha}\left(\left(0, H_{j}\right], D\right)} \leq \sigma, \quad 1 \leq j \leq J,
$$

where $H_{j}=T_{j}-T_{j-1}$ and $u_{T_{j}}(t)=u\left(T_{j-1}+t\right), 0 \leq t \leq H_{j}$.

Proof. Choose $R>0$ and $L>0$ as in (2.2) for $u^{*}=u(0)$. From the proof of Theorem 8.1.1 in [12, it follows that there exist constants $C>0$ and $0<H_{1} \leq T$ such that

$$
\begin{aligned}
& 2\|u-u(0)\|_{L^{\infty}\left(\left[0, H_{1}\right], D\right)}+\|u\|_{C_{\alpha}^{\alpha}\left(\left(0, H_{1}\right], D\right)} \\
& \quad \leq C\left\|\left(\mathrm{e}^{(\cdot) A}-1\right)(A u(0)+f(u(0)))\right\|_{C_{\alpha}^{\alpha}\left(\left(0, H_{1}\right], X\right)} .
\end{aligned}
$$

The constant $C$ depends on $L$ and the bound is valid as long as $\|u(t)-u(0)\|_{D} \leq R$ for $0 \leq t \leq H_{1}$. The right-hand side of (5.14) tends to 0 as $H_{1}$ goes to 0 . Thus, after a possible reduction of $H_{1}$, we get (5.13) with $j=1$. We go on with this construction, and since the constants $L$ and $R$ can be taken uniformly, the final time $T$ is reached after a finite number of steps.

\section{REFERENCES}

1. G. Akrivis, M. Crouzeix And C. Makridakis, Implicit-explicit multistep methods for quasilinear parabolic equations. Numer. Math. 82 (1999), 521-541. MR 2000e:65075

2. N. BAKAEV, On variable stepsize Runge-Kutta approximations of a Cauchy problem for the evolution equation. BIT 38 (1998), 462-485. MR 99i:65069

3. A. Belleni-Morante And A.C. McBride, Applied Nonlinear Semigroups. An Introduction. John Wiley \& Sons, Chichester, 1998. MR 99m:47083

4. K. Eriksson, C. Johnson and S. Larsson, Adaptative finite element methods for parabolic problems VI: Analytic semigroups. SIAM J. Numer. Anal. 35 (1998), 1315-1325. MR 99d:65281

5. C. González and C. Palencia, Stability of Runge-Kutta methods for quasilinear parabolic problems. Math. Comp. 69 (2000), 609-628. MR 2000i:65130

6. E. Hairer And M. Zennaro, On error growth functions of Runge-Kutta methods. Appl. Numer. Math. 22 (1996), 205-216. MR 97j:65116 
7. D. Henry, Geometric Theory of Semilinear Parabolic Equations. LNM 840, Springer, 1981. MR 83j:35084

8. M.-N. Le Roux, Méthodes multipas pour des équations paraboliques non linéaires. Numer. Math. 35 (1980), 143-162. MR 81i:65075

9. Ch. Lubich And A. Ostermann, Runge-Kutta methods for parabolic equations and convolution quadrature. Math. Comp. 60 (1993), 105-131. MR 93d:65082

10. Ch. Lubich And A. Ostermann, Runge-Kutta approximation of quasilinear parabolic equations. Math. Comp. 64 (1995), 601-627. MR 95g:65122

11. Ch. Lubich and A. Ostermann, Linearly implicit time discretization of non-linear parabolic equations. IMA J. Numer. Anal. 15 (1995), 555-583. MR 96g:65085

12. A. LunArdi, Analytic Semigroups and Optimal Regularity in Parabolic Problems. Birkhäuser, Basel, 1995. MR 96e:47039

13. E. Nakaguchi AND A. Yagi, Error estimates of implicit Runge-Kutta methods for quasilinear abstract equations of parabolic type. Japan. J. Math. (N.S.) 25 (1999), 181-226. MR 2000d:65164

14. C. PAlencia, On the stability of variable stepsize rational approximations of holomorphic semigroups. Math. Comp. 62 (1994), 93-103. MR 94c:47066

15. A. PAZY, Semigroups of Linear Operators and Applications to Partial Differential Equations. Springer, New York, 1983. MR 85g:47061

16. M.E. TAYlor, Partial Differential Equations III. Nonlinear Equations. Springer, New York, 1996. MR 98k:35001

17. M. Thalhammer, Runge-Kutta time discretization of nonlinear parabolic equations. Thesis, Universität Innsbruck, 2000.

Departamento de Matemática Aplicada y Computación, Facultad de Ciencias, UniVersidad de Valladolid, E-47011 Valladolid, Spain

E-mail address: cesareo@mac.cie.uva.es

Institut für Technische Mathematik, Geometrie und Bauinformatik, Universität Innsbruck, Technikerstrasse 13, A-6020 Innsbruck, Austria

Current address: Section de mathématiques, Université de Genève, rue du Lièvre 2-4, CH-1211

Genève 24, Switzerland

E-mail address: Alexander.Ostermann@uibk.ac.at, Alexander.Ostermann@math.unige.ch

Departamento de Matemática Aplicada y Computación, Universidad de Valladolid, Facultad de Ciencias, E-47011 Valladolid, Spain

E-mail address: palencia@mac.cie.uva.es

Institut für Technische Mathematik, Geometrie und Bauinformatik, Universität Innsbruck, Technikerstrasse 13, A-6020 Innsbruck, Austria

E-mail address: Mechthild.Thalhammer@uibk.ac.at 